\title{
Mechanisms of reactivation of latent tuberculosis infection due to SIV coinfection
}

\author{
Allison N. Bucşan, ${ }^{1}$ Ayan Chatterjee, ${ }^{2}$ Dhiraj K. Singh, ${ }^{2}$ Taylor W. Foreman, ${ }^{1}$ Tae-Hyung Lee, ${ }^{2}$ Breanna Threeton, \\ Melanie G. Kirkpatrick, ${ }^{1}$ Mushtaq Ahmed, ${ }^{3}$ Nadia Golden, ${ }^{1}$ Xavier Alvarez, ${ }^{1}$ James A. Hoxie, ${ }^{4}$ Smriti Mehra, ${ }^{1}$ \\ Jyothi Rengarajan,, ${ }^{5,6}$ Shabaana A. Khader, ${ }^{3}$ and Deepak Kaushal ${ }^{1,2}$
}

${ }^{1}$ Tulane National Primate Research Center, Covington, Louisiana, USA. ${ }^{2}$ Southwest National Primate Research Center, Texas Biomedical Research Institute, San Antonio, Texas, USA. ${ }^{3}$ Department of Molecular Microbiology, Washington University in St. Louis School of Medicine, St. Louis, Missouri, USA. ${ }^{4}$ Hematology-Oncology Division, University of Pennsylvania Perelman School of Medicine, Philadelphia, Pennsylvania, USA. ${ }^{5}$ Emory Vaccine Center and ${ }^{6}$ Department of Medicine, Emory University, Atlanta, Ceorgia, USA.

\begin{abstract}
HIV is a major driver of tuberculosis (TB) reactivation. Depletion of CD4 ${ }^{+} \mathrm{T}$ cells is assumed to be the basis behind TB reactivation in individuals with latent tuberculosis infection (LTBI) coinfected with HIV. Nonhuman primates (NHPs) coinfected with a mutant simian immunodeficiency virus (SIV $\Delta G Y$ ) that does not cause depletion of tissue CD4+ T cells during infection failed to reactivate TB. To investigate the contribution of $\mathrm{CD}^{+} \mathrm{T}$ cell depletion relative to other mechanisms of SIVinduced reactivation of LTBI, we used CD4R1 antibody to deplete CD4+ $T$ cells in animals with LTBI without lentiviral infection. The mere depletion of CD4+ $T$ cells during LTBI was insufficient in generating reactivation of LTBI. Instead, direct cytopathic effects of SIV resulting in chronic immune activation, along with the altered effector $T$ cell phenotypes and dysregulated $T$ cell homeostasis, were likely mediators of reactivation of LTBI. These results revealed important implications for TB control in HIV-coinfected individuals.
\end{abstract}

\section{Introduction}

Tuberculosis (TB) and HIV coinfection are a global syndemic (1). Approximately $90 \%$ of HIV-negative Mycobacterium tuberculosis-infected ( $M t b$-infected) individuals lack clinical signs of TB and are considered to have latent TB infection (LTBI) $(1,2)$. Although the immune systems of exposed individuals successfully contain $M t b$ infection in most cases, bacteria can persist within lung granulomas for long periods before reactivating to TB disease $(3,4)$. We seek to understand the mechanisms by which HIV coinfection reactivates TB using the $M t b /$ SIV coinfection model in rhesus macaques.

$\mathrm{CD}^{+} \mathrm{T}$ cell depletion is a hallmark of HIV and SIV infection, and is widely regarded as the primary cause of immunodeficiency. Previously, we identified $M t b / S I V-c o i n f e c t e d$ primates that did not progress to TB despite productive coinfection, raising the possibility of $\mathrm{CD}^{+} \mathrm{T}$ cell-independent mechanisms of LTBI control (5). To investigate the contribution of $\mathrm{CD}^{+} \mathrm{T}$ cell depletion relative to other mechanisms of SIV-induced reactivation of LTBI, we used antibodies to deplete $\mathrm{CD} 4^{+} \mathrm{T}$ cells and compared the outcomes to coinfection with pathogenic SIV and the mutant virus, SIV $\Delta G Y$. Coinfection of $M t b$ with pathogenic SIV, but without mutant or antibody-mediated $\mathrm{CD}^{+} \mathrm{T}$ cell depletion, resulted in reactivation.

Conflict of interest: The authors have declared that no conflict of interest exists. Copyright: (c) 2019, American Society for Clinical Investigation.

Submitted: February 19, 2019; Accepted: August 29, 2019; Published: October 28, 2019. Reference information: J Clin Invest. 2019;129(12):5254-5260.

https://doi.org/10.1172/JCl125810.

\section{Results and Discussion}

To assess the role of lung $\mathrm{CD} 4^{+} \mathrm{T}$ cells in protecting against reactivation of LTBI, 39 Indian rhesus macaques were exposed to lowdose aerosol Mycobacterium tuberculosis (Mtb) infection to establish TB latency (Figure 1A and Supplemental Table 1; supplemental material available online with this article; https://doi.org/10.1172/ JCI125810DS1). At week 9 after TB infection, a subset of 16 macaques experiencing asymptomatic LTBI were coinfected with pathogenic SIVmac239. These animals exhibited 2 distinct outcomes: 9 animals developed signs of reactivated TB (reactivators) while 7 retained $M t b$ latency despite productive SIV infection and peripheral blood viremia (nonreactivators) (5). To investigate the role of $\mathrm{CD}^{+} \mathrm{T}$ cells in our low-dose aerosol model, we coinfected 6 macaques with a novel variant of pathogenic SIVmac29 molecular clone, SIVmac239 $\Delta$ GY (SIV $\Delta G Y)$ (6), in which a deletion of 2 amino acids from a trafficking motif in the envelope gp41 cytoplasmic domain leads to viral replication, but does not deplete CD $4^{+}$ $\mathrm{T}$ cells in the periphery or in the lamina propria (ref. 7 and Supplemental Table 1). In addition, we used antibody-mediated depletion of $\mathrm{CD}^{+} \mathrm{T}$ cells in 8 macaques with LTBI using CD4R1, which was administered every 2 weeks starting at week 9 after $M t b$ infection (Figure 1A and Supplemental Table 1).

Importantly, SIV $\Delta$ GY-coinfected and CD4R1-administered macaques retained control of TB similar to nonreactivators. Specifically, only 1 of 8 CD4R1-administered nonhuman primates (NHPs) displayed symptomatology consistent with reactivated TB that necessitated a humane necropsy (Figure 1). SIV $\Delta G Y-$ coinfected and CD4R1-administered macaques showed normal serum C-reactive protein (CRP) levels over time (Supplemen- 
A

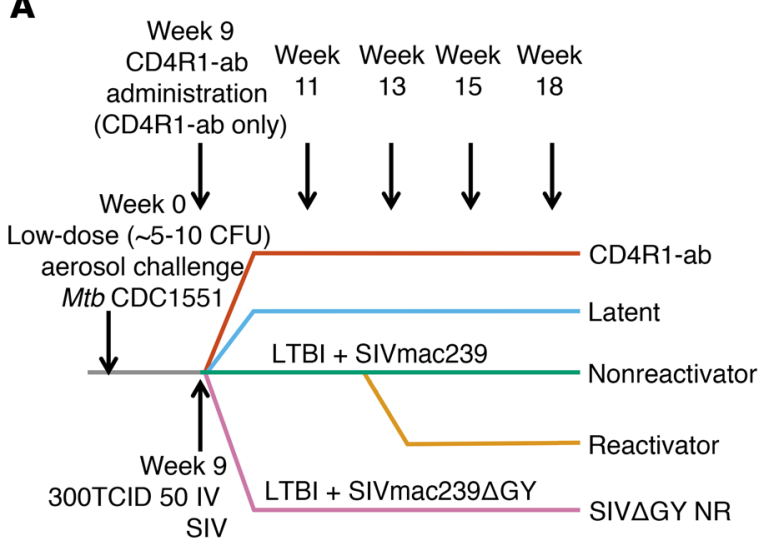

B

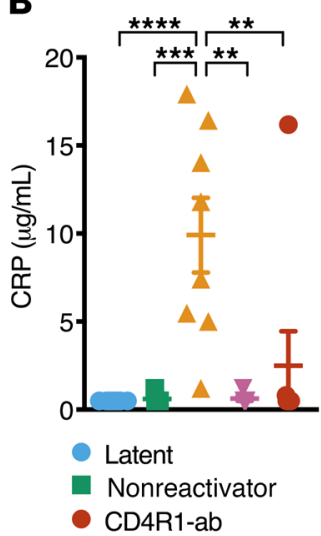

C

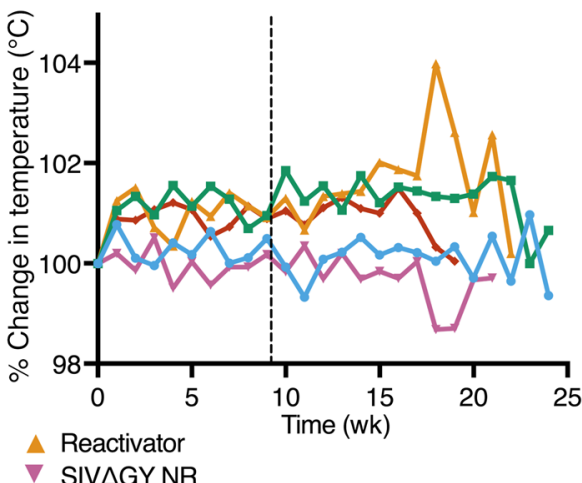

D

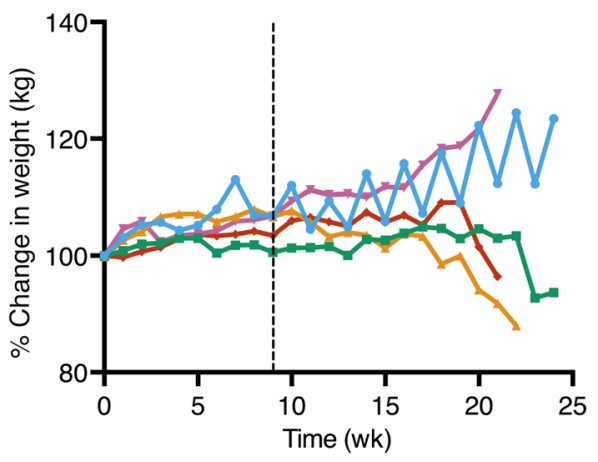

G

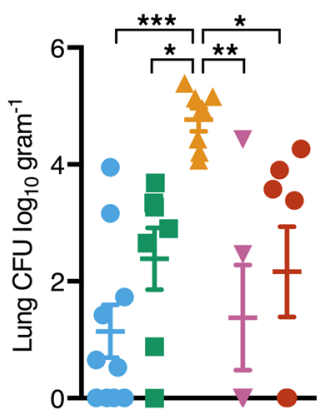

H

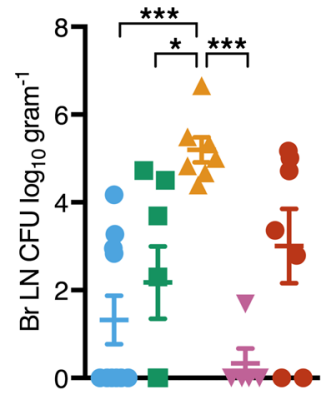

E

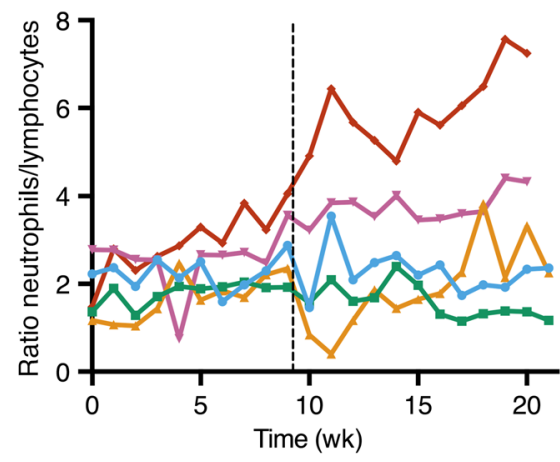

I

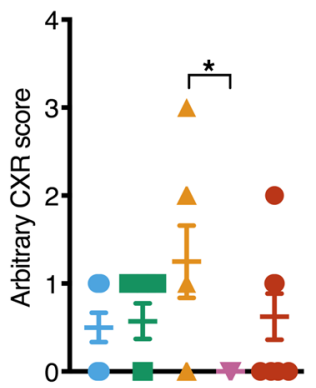

\section{J}

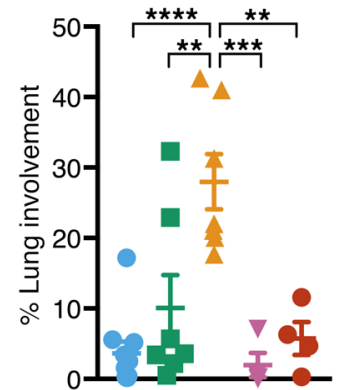

$\mathbf{F}$

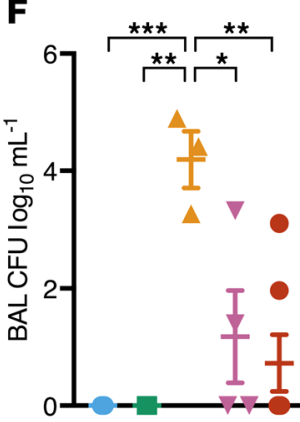

Figure 1. Comparison of CD4 ${ }^{+} \mathrm{T}$ cell-sparing SIVmac239 $\Delta \mathrm{GY}$ and antibody-mediated CD4 ${ }^{+} \mathrm{T}$ cell depletion using CD4R1 in Mtb-infected NHPs. (A) Study outline. (B) Serum CRP levels at necropsy. (C) Percentage of temperature increase $\left({ }^{\circ} \mathrm{C}\right)$ and $(\mathbf{D})$ percentage of weight loss $(\mathrm{kg})$ compared with baseline. $(\mathrm{E})$ Ratio of neutrophils to lymphocytes in clinical blood. Bacterial burden at necropsy measured in (F) BAL, (C) lung, and (H) bronchial lymph nodes. (I) CXR scores established by veterinary clinicians for pneumonia. (J) Percentage of lung involvement determined by board-certified veterinary pathologists. (K) Peak plasma viral loads (PVLs) measured at peak. ${ }^{*} P<0.05 ;{ }^{* *} P<0.01 ;{ }^{* *} P<0.001 ;{ }^{* * *} P<0.0001$, 1-way ANOVA with Tukey's multiple testing correction. C-E represent mean, and $\mathbf{B}$ and $\mathbf{F}-\mathbf{J}$ represent mean \pm SEM.

tal Figure 1A) and at endpoint (Figure 1B), comparable to LTBI and nonreactivators and statistically different from reactivators. These animals maintained relatively normal body temperatures (Figure 1C) and weights (Figure 1D). Reactivators, unlike all other groups, had a lower ratio of neutrophils/lymphocytes after SIV coinfection at week 9 (Figure 1E). SIV $\Delta$ GY-coinfected and CD4R1-administered NHPs had lower numbers of viable $M t b$ in their bronchoalveolar lavage (BAL) fluid throughout infection (Supplemental Figure 1B), and significantly lower viable $M t b$ in their BAL at endpoint (Figure $1 \mathrm{~F}$ ). Similarly, both experimental groups harbored low lung (Figure $1 \mathrm{G}$ ), bronchial lymph node (Figure 1H), spleen (Supplemental Figure 1C), liver (Supplemental Figure 1D), and kidney (Supplemental Figure 1E) bacterial burdens, comparable to the LTBI and nonreactivators. Both experimental groups possessed significantly lower viable $M t b$ in all tissues at necropsy compared with reactivators. Finally, virtually no tuberculous lung pathology was observed in SIV $\Delta$ GY-coinfected NHPs, demonstrating that coinfection with this virus failed to reactivate LTBI (Figure 1, I and J). One of 8 CD4R1-administered NHPs with LTBI did reactivate, displaying an elevated CRP at necropsy (Figure 1B) and chest X-ray (CXR) score (Figure 1I). Measurement of peripheral viremia in coinfected animals suggested that 
A
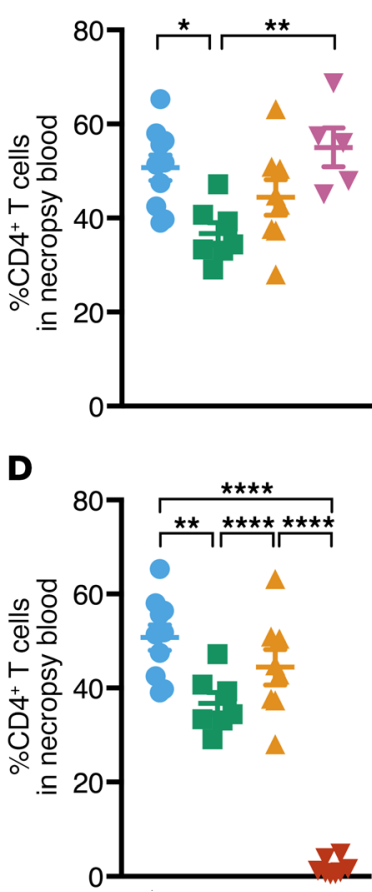

B

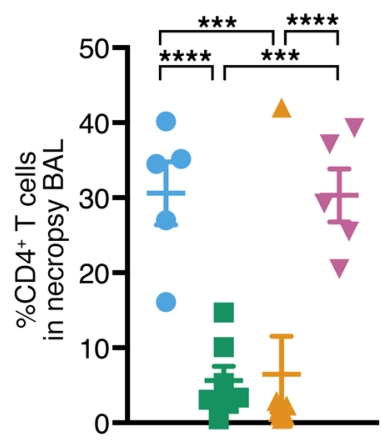

E

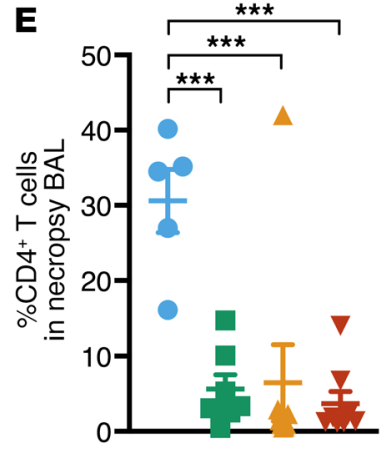

C
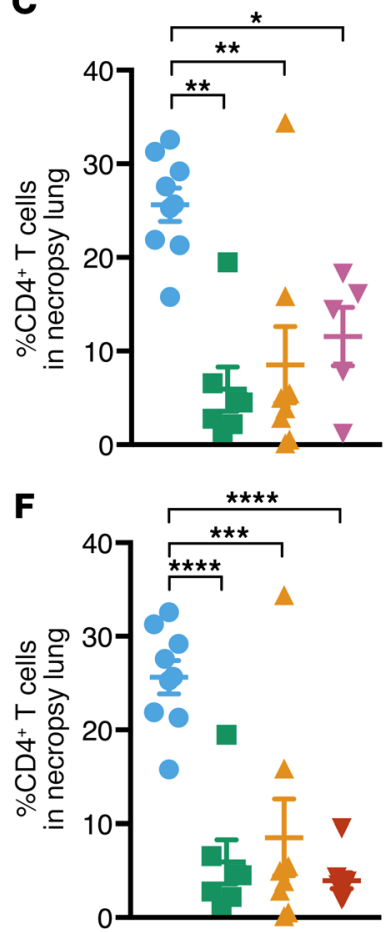

Reactivator

$\checkmark$ SIV $\triangle G Y N R$
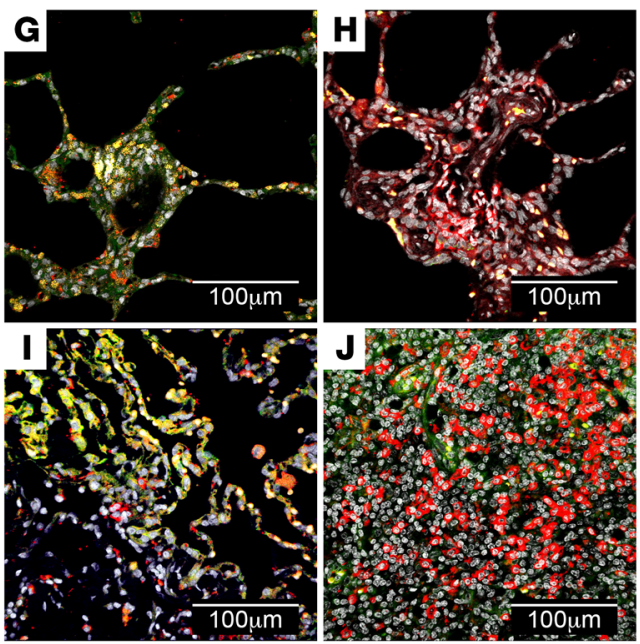

Latent

Nonreactivator

- CD4R1-ab but to spare gut mucosal tissues $(7,8)$. To our knowledge, this was a first-time evaluation of lung $\mathrm{CD}^{+} \mathrm{T}$ cell populations in SIV $\Delta G Y$-infected NHPs, so this was a novel finding. There may be sufficient lymphoid tissue in the lungs that $\operatorname{SIV} \Delta G Y$ was able to replicate nearby, perhaps in inducible bronchusassociated lymphoid tissue (iBALT) (5), which led to $\mathrm{CD}^{+} \mathrm{T}$ cell depletion during SIV $\triangle G Y$ coinfection. Significant depletion of $\mathrm{CD} 4^{+} \mathrm{T}$ cells was observed in the peripheral blood (Figure 2D), BAL (Figure 2E), and lungs (Figure 2F) of CD4R1-administered NHPs, relative to LTBI NHPs. The levels of $\mathrm{CD}^{+}{ }^{+} \mathrm{T}$ cell depletion were comparable to SIVmac239-infected animals and the differences in $\mathrm{CD} 4^{+} \mathrm{T}$ cell populations between NHPs administered CD4R1 and NHPs coinfected with SIVmac239 were not significant in the BAL or lungs (Figure 2, E and
SIV $\Delta G Y$ replicated to comparable levels in the acute phase and established similar set points (Figure $1 \mathrm{~K}$ ). Although significantly lower peripheral viremia was observed at peak in our SIV $\Delta$ GY-coinfected NHPs compared with SIVmac239-coinfected reactivators and nonreactivators, this is not unexpected as rhesus macaques infected with SIV $\Delta G Y$ often have variable viremia $(8,9)$.

NHPs with LTBI/SIV $\Delta$ GY coinfection did not exhibit a significant decline in $\mathrm{CD}^{+} \mathrm{T}$ cell levels in peripheral blood (Figure $2 \mathrm{~A}$ and Supplemental Figure 2, A and C) or BAL (Figure 2B and Supplemental Figure 2, B and D). This was in stark contrast to animals infected with pathogenic SIV (Figure 2, A and B), consistent with previous results $(5,10)$. Although a significant reduction in $\mathrm{CD} 4^{+} \mathrm{T}$ cells was observed in the lungs (Figure $2 \mathrm{C}$ ) of SIV $\Delta \mathrm{GY}$-coinfected NHPs, an insignificant reduction was observed in the total CD $4^{+} \mathrm{T}$ cell compartment (Supplemental Figure 2E). Previously, SIV $\Delta G Y$ had been shown to replicate in the plasma and lymphoid tissues,
F), regardless of their TB outcome. This result was confirmed by multilabel confocal immunofluorescence microscopy (Figure 2, $\mathrm{G}-\mathrm{K})$. Thus, our results show that coinfection of macaques with LTBI with a nonpathogenic virus that fails to deplete CD $4^{+} \mathrm{T}$ cells (SIV $\Delta \mathrm{GY}$ ) and depletion of $\mathrm{CD} 4^{+} \mathrm{T}$ cells using antibodies does not result in similar rates of $\mathrm{TB}$ reactivation observed upon coinfection with pathogenic SIVmac239.

As early as week 2 after SIV challenge or CD4R1 administration, NHPs displayed significant changes in the distribution of their residual $\mathrm{CD}^{+} \mathrm{T}$ cells. $\mathrm{M} t b$ infection alone can impact the distribution of effector and memory cells, and memory $\mathrm{CD} 4^{+} \mathrm{T}$ cells are associated with protection (11-13). We compared 4 experimental groups of NHPs: our SIVmac239-coinfection reactivators and nonreactivators, SIV $\Delta G Y$-coinfected, and CD4R1-administered NHPs. Although $\mathrm{CD}^{+} \mathrm{T}$ cells were massively depleted in both experimental groups of NHPs, it was vital 
A

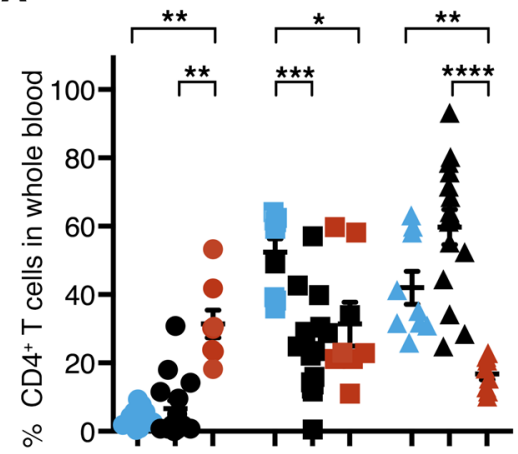

D

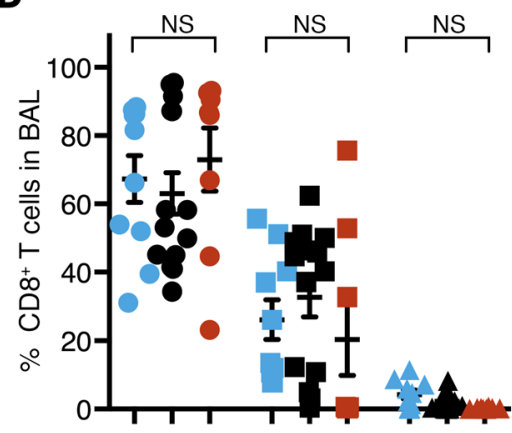

G

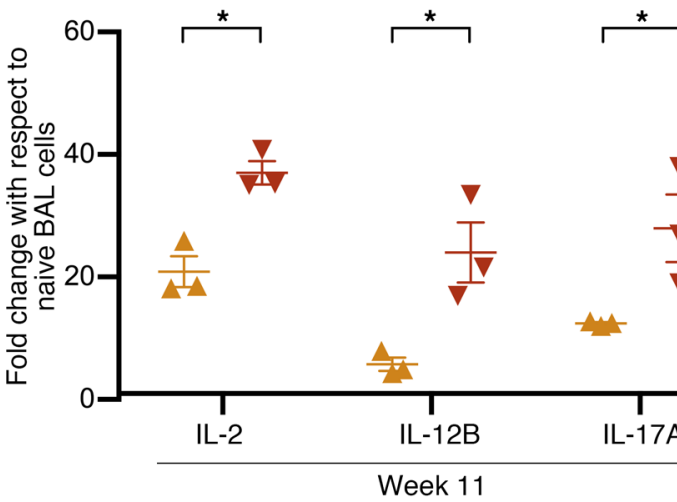

B

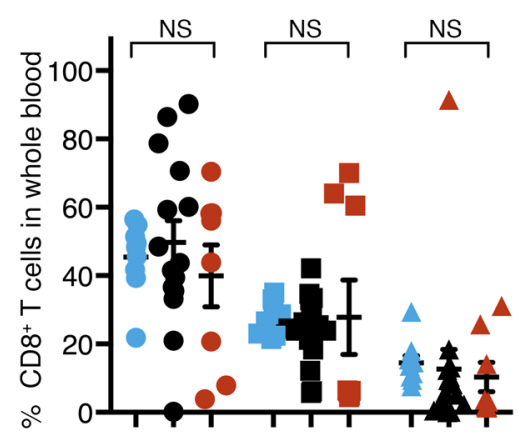

E

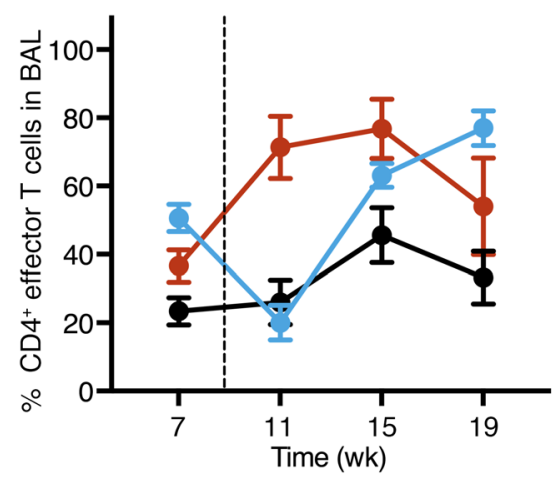

C

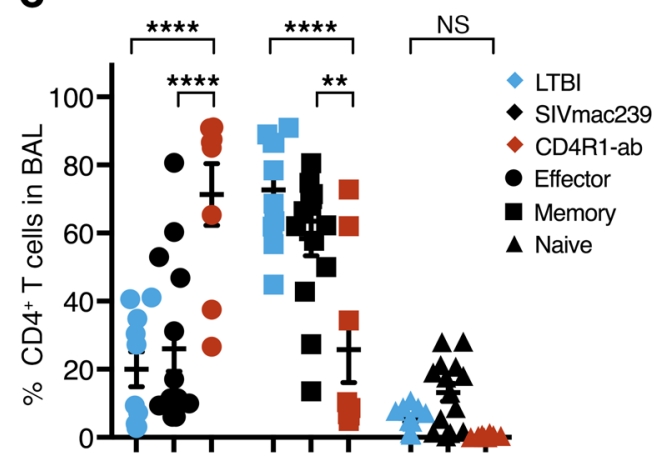

$\mathbf{F}$

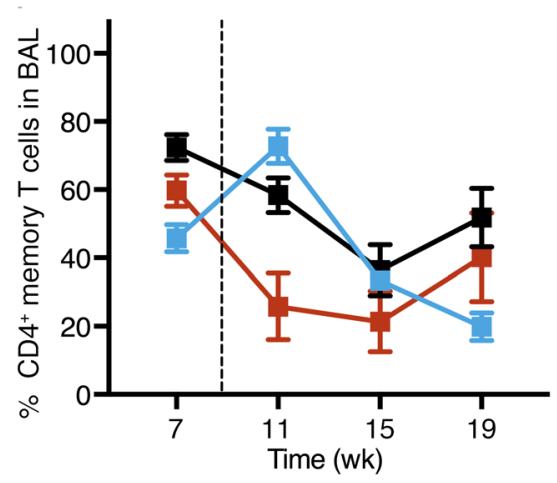

Reactivator $\nabla \mathrm{CD} 4 \mathrm{R} 1-\mathrm{ab}$

Figure 3. Peripheral and BAL CD4 ${ }^{+}$cells reflect a proportional shift toward effector profile during CD4R1 administration. The proportions of residual effector (CD95 ${ }^{+} \mathrm{CD28} 8^{-}$, circles), memory (CD95 ${ }^{+} \mathrm{CD} 28^{+}$, squares), and naive (CD95-CD28+, triangles) (A) CD4+ and (B) CD8 ${ }^{+}$T cells in the peripheral blood and (C) CD4 ${ }^{+}$and (D) CD8 ${ }^{+}$T cells in the BAL of SIVmac239-coinfected $(n=15$, black) and CD4R1-administered NHPs $(n=8$, red) compared with LTBI NHPs $(n=$ 10, blue) at week 11 after TB. ${ }^{*} P<0.05$; ${ }^{*} P<0.01$; ${ }^{* *} P<0.001$; ${ }^{* * *} P<0.0001$, 2-way ANOVA with Tukey's multiple testing correction. BAL (E) effector and (F) memory CD4+ cells quantification every 4 weeks starting at week 7 after TB. Coinfection and antibody administration shown by the dotted line. (G) qRT-PCR analysis of IL-2, IL-12B, and IL-17A collected from BAL cells at week 11 and week 15 after TB from SIV reactivators ( $n=3$, yellow) and CD4R1-administered NHPs $\left(n=3\right.$, red). ${ }^{*} P<0.05 ;{ }^{* *} P<0.01 ;{ }^{* *} P<0.001$; ${ }^{* * *} P<0.0001$, multiple $t$ tests with Holm-Sidak method for multiple comparison correction. Data represent mean $\pm \mathrm{SEM}$.

to understand the contribution of the remaining $\mathrm{CD} 4^{+} \mathrm{T}$ cells to the host's immune defenses. Coinfection with SIVmac239 resulted in a significant reduction of memory $\left(\mathrm{CD} 95^{+} \mathrm{CD} 28^{+}\right)$ $\mathrm{CD}^{+} \mathrm{T}$ cells, which were replaced by naive $\left(\mathrm{CD}^{-} 5^{-} \mathrm{CD} 28^{+}\right)$, not effector $\left(\mathrm{CD}^{+} 5^{+} \mathrm{CD} 28^{-}\right), \mathrm{CD}^{+} \mathrm{T}$ cells (Figure $\left.3 \mathrm{~A}\right)$. Most remaining $\mathrm{CD}^{+}{ }^{+} \mathrm{T}$ cells in the blood of SIV-coinfected animals were naive, consistent with findings in human studies that $M t b$ / HIV-coinfected patients harbor less differentiated $\mathrm{CD}^{+}$memory T cells compared with TB-only patients (14). HIV infection can lead to an activated and exhausted memory $\mathrm{CD} 4^{+} \mathrm{T}$ cell phenotype (15), which likely contributes to the more significant loss of memory $\mathrm{CD} 4^{+} \mathrm{T}$ cells. Compared with animals with LTBI, CD4R1-administered NHPs exhibited significantly higher frequencies of effector and reduced naive and memory $\mathrm{CD} 4^{+} \mathrm{T}$ cells in the peripheral blood (Figure 3A). In the peripheral blood, $\mathrm{CD}^{+} \mathrm{T}$ cells did not differ between groups (Figure $3 \mathrm{~B}$ ). In the $\mathrm{BAL}$, changes were observed only in the $\mathrm{CD} 4^{+}$(Figure $3 \mathrm{C}$ ) and not the $\mathrm{CD}^{+} \mathrm{T}$ cell compartment (Figure 3D). Memory $\mathrm{CD} 4^{+} \mathrm{T}$ 

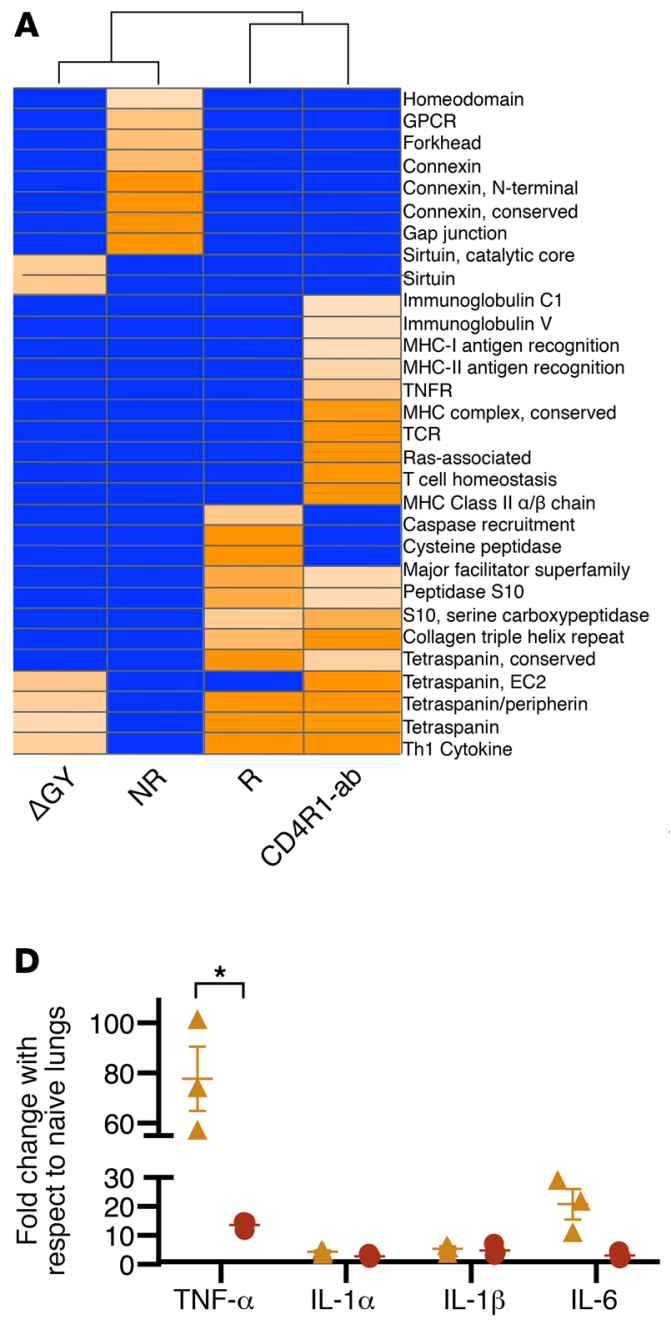

$\mathbf{F}$

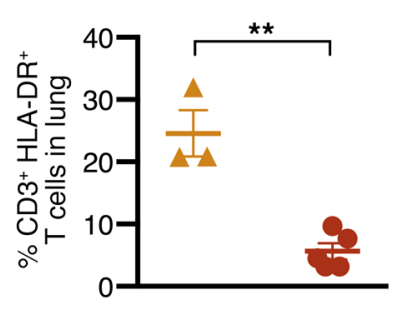

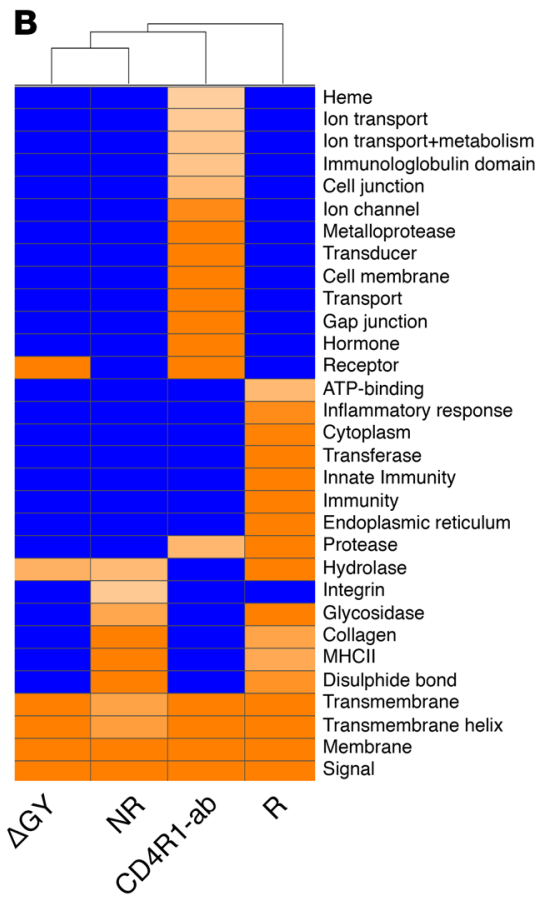

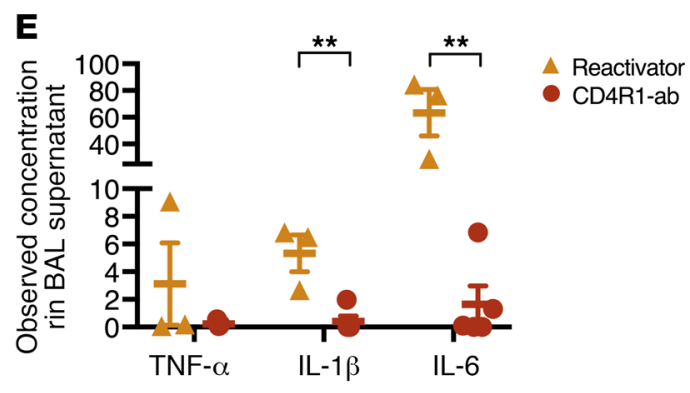

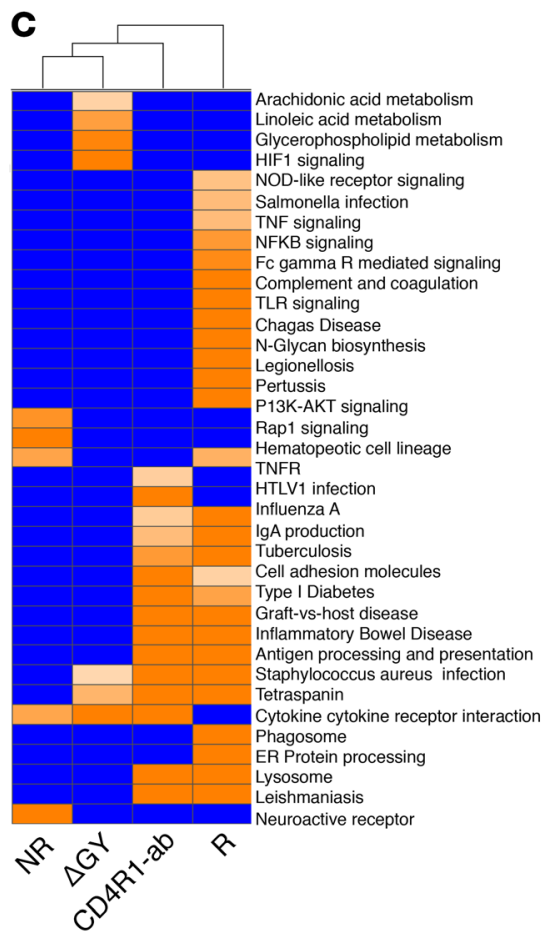

H
G

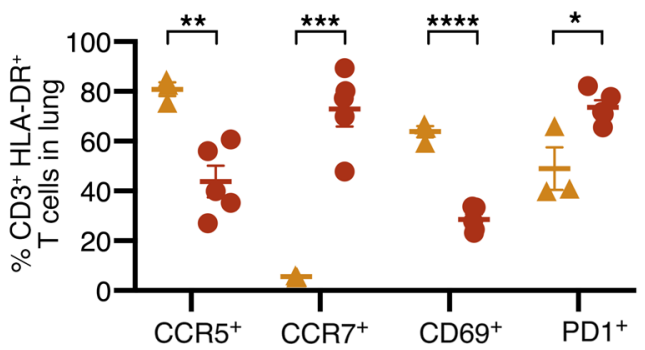

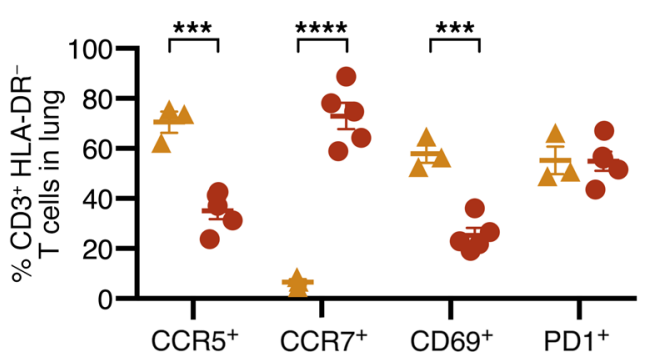

Figure 4. Immunological analyses of Mtb/SIV-coinfected and CD4R1-administered NHPs reveal reactivation-associated immune impairment. Transcriptomic results generated for necropsy lung tissue ( $n=3$ per group) using (A) InterPro, (B) DAVID Enriched Categories, and (C) KEGC Enriched Pathways. (D) qRT-PCR analysis of TNF- $\alpha$, IL-1 $\alpha$, IL-1 $\beta$, and IL- 6 from necropsy lung cells of SIV reactivators ( $n=3$, yellow) and CD4R1-administered NHPs ( $n=3$, red). (E) The concentration of TNF- $\alpha$, IL-1 $\beta$, and IL-6 measured in the necropsy BAL supernatant of SIV reactivators ( $n=3$, yellow) and CD4R1-administered NHPs ( $n$ $=5$, red). (F) T cell expression of HLA-DR in necropsy lung and coexpression of migratory (CCR5 and CCR7), activation (CD69), and apoptotic (PD-1) markers measured in the (G) HLA-DR+ and (H) HLA-DR ${ }^{-}$T cell population. ${ }^{*} P<0.05 ;{ }^{* *} P<0.01 ;{ }^{* *} P<0.001 ;{ }^{* * *} P<0.0001$. Unpaired, 2-tailed $t$ test used for $\mathbf{F}$, and multiple, unpaired $t$ tests used for $\mathbf{G}$ and $\mathbf{H}$. Data represent mean $\pm \mathrm{SEM}$.

cells were replaced by naive cells upon SIV coinfection in the BAL (Figure 3C). Interestingly, the distribution of BAL CD4 ${ }^{+}$ $\mathrm{T}$ cells following $\mathrm{CD} 4^{+} \mathrm{T}$ cell depletion by SIVmac239 coinfection did not significantly differ from the distributions observed during LTBI, indicating that the virus affected all compartments equally within the BAL. BAL effector $\mathrm{CD} 4^{+} \mathrm{T}$ cell populations were significantly increased in CD4R1-administered NHPs compared with both LTBI and SIVmac239-coinfected NHPs, whereas memory $\mathrm{CD} 4^{+} \mathrm{T}$ cell populations were significantly reduced (Figure 3C). Throughout CD4R1 administration, $\mathrm{CD}^{+} \mathrm{T}$ cells in the BAL reflected a bias toward effector (Figure $3 \mathrm{E}$ ) rather than memory (Figure 3F) populations. Greater proportions of effector $\mathrm{T}$ cells in the lungs of CD4R1-administered, versus SIV-coinfected, NHPs strongly suggest that early 
pathogenic events associated with SIV coinfection suppress the natural homeostatically driven $\mathrm{CD}^{+} \mathrm{T}$ cell proliferation and downstream effector $\mathrm{T}$ cell functions. As these differences were unique to the BAL and not the lung tissue (Supplemental Figure 3 , A and B), they suggest that $\mathrm{T}$ cells in the lung, and not circulating BAL cells, are capable of retaining their typical functions.

Gene-expression analyses in the BAL from SIV-coinfected versus CD4R1-administered NHPs confirmed our assertion that $\mathrm{CD}^{+}{ }^{+} \mathrm{T}$ cell maturation states differed in these 2 groups. Real-time RT-PCR analysis revealed that at week 11 and 15 after $M t b$, NHPs administered CD4R1 had significantly higher levels of transcripts involved in $\mathrm{T}$ cell activation, differentiation, and function (e.g., IL-2, key signal for Th1 differentiation; IL-12B, important for sustaining memory/effector Th1 responses against $M t b$; and IL-17A, expressed on $\mathrm{Th} 17^{+}$cells) compared with SIV reactivators (Figure $3 G)$. These cytokines indicate that although CD4R1 depleted the $\mathrm{CD}^{+} \mathrm{T}$ cell compartment, it did not inhibit $\mathrm{T}$ cell maturation and signaling. On the contrary, SIV coinfection inhibited T cell maturation and signaling.

Gene-enrichment analyses on lung transcriptome confirmed that SIV coinfected reactivators experienced chronic immune activation and a cytokine storm. $\mathrm{CD} 4^{+} \mathrm{T}$ cell homeostasis was enhanced in CD4R1-administered NHPs and unperturbed during SIV coinfection. The gene terms "immunoglobulin/major histocompatibility complex (MHC)," "MHC class I/class II antigen recognition protein," etc., were significantly enriched only in CD4R1-administered NHPs (Figure 4A and Supplemental Table 2, InterPro). Several categories encoding proteins interacting with RAS (Figure 4A), involved in cell signaling pathways that control cell growth and cell death, were only significantly enriched in CD4R1-administered NHPs. This underscores that normal immune homeostasis is observed in CD4R1-administered NHPs, despite severe lymphopenia. Categories such as "inflammatory response," "innate immunity," and "endoplasmic reticulum" were only enriched in SIV reactivators, suggesting chronic immune activation and an ensuing cytokine storm in that group (Figure 4B). Unsurprisingly, SIV reactivators were characterized by the enrichment of gene categories corresponding to chronic immune activation and inflammation, including "TNF-signaling" and "Fc-gamma receptor mediated phagocytosis" (Figure 4C). Reactivator lungs substantially expressed high levels of genes involved in proinflammatory cytokine/chemokine signaling (CXCL12, IL6), myeloid cell/phagocyte/complement/innate immune signaling (CD68, $C F H, C 1 S$, TXNDC11), matrix degradation critical for granuloma formation (IGFBP7), and antigen presentation ( $H L A B)$ (Supplemental Table 2). Genes belonging to the pathway "regulation of lymphocyte differentiation," including NDFIP1, $F A S$, and RNF41, were induced in lungs of reactivators. The genes only expressed in nonreactivators' lungs included those involved in the restoration of normal lung and cytoskeletal function (WWOX, TRIOBP, PEAK3), B cell function (SPANXA1) (16), repair of acute tissue injury (MYLK2) (17), inhibition of cytokine storm (TIP39, SLC8A2) (18), viral restriction (APOBEC3) (19), induction of apoptosis (IFT57), and induction of robust cellular immune responses (CLECB15/KLRG2, BTN2A3, TSPEAR, MRAP).
Real-time RT-PCR and multiplex cytokine analysis strongly confirmed genome-wide transcriptome results. Cytokines associated with inflammation, including $T N F-\alpha, I L 1-\alpha, I L-1 \beta$, and $I L-6$, were highly induced in the lungs of SIV-coinfected reactivators compared with CD4R1-administered NHPs (Figure 4D). Multiplex analysis supported this trend, showing that in the BAL supernatant at necropsy, TNF- $\alpha$, IL1- $\alpha$, IL-1 $\beta$, and IL- 6 proteins were more abundant in SIV-coinfected reactivators (Figure 4E). Significantly increased IFN- $\gamma$ was detected in the plasma (Supplemental Figure 4A) and BAL (Supplemental Figure 4B) of reactivators compared with other groups. Other markers of increased cell signaling and migration associated with inflammatory responses were significantly increased in the plasma (CXCL9; Supplemental Figure 4C) or BAL (CXCL13 [Supplemental Figure 4D], eotaxin [Supplemental Figure 4E], I-TAC [Supplemental Figure 4F], and IL-6 [Supplemental Figure $4 G]$ ) of reactivators. SIV $\Delta G Y$-coinfected NHPs had significantly increased IL-8 and MIP1- $\beta$ in the plasma (Supplemental Figure 4, H and I) compared with all other groups, and LTBI NHPs had significantly increased eotaxin in the plasma (Supplemental Figure 4J) and SDF- $1 \alpha$ in BAL (Supplemental Figure $4 \mathrm{~K}$ ).

Chronic immune activation in SIV-coinfected animals was supported by immunophenotyping. T cells in the lungs of reactivators had a significantly more activated phenotype (HLA-DR $\left.{ }^{+}\right)$ compared with those from NHPs that received CD4R1 (Figure 4F). HLA-DR ${ }^{+}$(Figure 4G) and HLA-DR (Figure 4H) T cells in reactivators exhibited significantly higher CCR5 (recruitment) and CD69 (early activation) markers compared with CD4R1 administration. This suggests higher recruitment and entrapment of activated lymphocytes at the primary site of infection in reactivators. CCR5-associated recruitment to the immune synapse induces stronger T cell-APC attraction, which results in reduced $\mathrm{T}$ cell responsiveness to chemokines (20). This response is typically followed by increased $\mathrm{T}$ cell proliferation and upregulated effector responses $(20,21)$, which was observed through increased CD69 expression by SIV reactivators. Furthermore, $\mathrm{CCR7}^{+} \mathrm{T}$ cell populations were significantly reduced in reactivators compared with CD4R1-administered NHPs (Figure 4, $\mathrm{G}$ and $\mathrm{H}$ ), suggesting that migration to secondary lymph nodes was impaired in T cells from the lungs of reactivators (22). Significantly reduced $\mathrm{PD}-1$ expression in reactivators compared with CD4R1-administered NHPs indicated that HLA-DR+ (Figure 4G), but not HLA-DR (Figure $4 \mathrm{H}$ ), T cells in reactivators were subject to regulation, strongly supporting a pattern of chronic immune activation in reactivators. As this pattern was also observed in the $\mathrm{CD}^{+} \mathrm{T}$ cell compartment (Supplemental Figure 5, A-C), SIV-mediated $\mathrm{CD} 4^{+} \mathrm{T}$ cell depletion is likely not the sole cause of immune imbalance leading to chronic activation, as T cell recruitment, activation, and homeostatic regulation was maintained in NHPs with antibody-mediated $\mathrm{CD} 4^{+} \mathrm{T}$ cell depletion. As can be seen in the reactivators with reduced PD-1 expression, some of these inhibitory responses are not being maintained, thus allowing increased immune activation and the cytokine storm seen in our transcriptomics data to occur.

Although it is known that the functions of mycobacterialspecific $\mathrm{CD}^{+}{ }^{+} \mathrm{T}$ cells are impaired during HIV infection, the immunological mechanisms underlying how $\mathrm{CD}^{+} \mathrm{T}$ cells contribute to maintaining immune control during LTBI and how HIV perturbs 
this control are not understood (23). Not all NHPs with LTBI/SIV coinfection exhibit reactivation despite comparable $\mathrm{CD} 4^{+} \mathrm{T}$ cell depletion (5), indicating that $\mathrm{CD}^{+} \mathrm{T}$ cell-independent mechanisms exist for the maintenance of LTBI control. Coinfection of animals with SIV $\Delta G Y$ showed that $\mathrm{CD} 4^{+} \mathrm{T}$ cells are important for preventing reactivation during $M t b / S I V$. However, the mere depletion of $\mathrm{CD}^{+} \mathrm{T}$ cells is insufficient for reactivation of LTBI, since only 1 of 8 CD4R1-administered NHPs developed overt TB disease. The key difference in the lungs of primates where lung $\mathrm{CD}^{+} \mathrm{T}$ cells were depleted by antibody or by pathogenic SIV was that the signatures of chronic immune activation and blockade of normal lymphocytic homeostasis were detected in the lungs of only reactivators.

Our results show that lung-specific chronic immune activation may be important for SIV-mediated (and perhaps HIVmediated) reactivation of LTBI. Since CD4R1 administration resulted in greater depletion of memory $\mathrm{CD} 4^{+} \mathrm{T}$ cells and preservation of effector $\mathrm{CD}^{+} \mathrm{T}$ cells, it is possible that this homeostasis drives the lack of reactivation in these NHPs. It is further acknowledged that the lack of chronic immune activation may be a byproduct of the corresponding lack of reactivation. Importantly, it appears that SIV pathogenicity drives mechanisms such as chronic immune activation that disturb the normal homeostasis of the effector $\mathrm{CD}^{+}$population. Understanding how SIV and chronic immune activation impact immunity to TB could lead to the development of new treatment paradigms aimed at the cure of $M t b / \mathrm{HIV}$ coinfection.

\section{Methods}

Study approval. All procedures were approved by the Tulane National Primate Research Center Institutional Animal Care and Use Committee and Tulane Institutional Biosafety Committee.

\section{Author contributions}

ANB, SAK, and DK designed the study. ANB, AC, TWF, XA, BT, MGK, and NG performed experiments. ANB, AC, TWF, XA, DKS, THL, SAK, and DK analyzed results. JAH, JR, SM, and MA interpreted results and provided vital reagents. ANB, JR, SM, MA, SAK, and DK prepared the manuscript.

\section{Acknowledgments}

This research was funded by NIH award AI111914 to DK and SAK, with additional support from awards AI134240, AI123047, AI123780, AI111943, OD011104, and OD011133. CD4R1 antibody provided by NHP Reagent Resource, Boston, MA, USA. Complete transcriptomics raw data can be retrieved from the Gene Expression Omnibus database (accession number GPL10183).

Address correspondence to: Deepak Kaushal, Southwest National Primate Research Center, Texas Biomedical Research Institute, 8715 West Military Drive, San Antonio, Texas 78227, USA. Phone: 210.258.9209; Email: dkaushal@txbiomed.org. Or to: Shabaana A. Khader, Washington University in St. Louis School of Medicine, 660 South Euclid Avenue, St. Louis, Missouri 63110, USA. Phone: 314.286.1590; Email: sakhader@wustl.edu.
1. WHO. Global tuberculosis report 2017. Geneva, Switzerland: WHO Press; 2017.

2. Kwan CK, Ernst JD. HIV and tuberculosis: a deadly human syndemic. Clin Microbiol Rev. 2011;24(2):351-376.

3. Lillebaek T, Dirksen A, Baess I, Strunge B, Thomsen VØ, Andersen AB. Molecular evidence of endogenous reactivation of Mycobacterium tuberculosis after 33 years of latent infection. J Infect Dis. 2002;185(3):401-404.

4. Lillebaek T, Dirksen A, Vynnycky E, Baess I, Thomsen VØ, Andersen AB. Stability of DNA patterns and evidence of Mycobacterium tuberculosis reactivation occurring decades after the initial infection. J Infect Dis. 2003;188(7):1032-1039.

5. Foreman TW, et al. CD4+ T-cell-independent mechanisms suppress reactivation of latent tuberculosis in a macaque model of HIV coinfection. Proc Natl Acad Sci US A. 2016;113(38):E5636-E5644.

6. Breed MW, et al. A single amino acid mutation in the envelope cytoplasmic tail restores the ability of an attenuated simian immunodeficiency virus mutant to deplete mucosal CD4+ T cells. J Virol. 2013;87(23):13048-13052.

7. Breed MW, et al. Loss of a tyrosine-dependent trafficking motif in the simian immunodeficiency virus envelope cytoplasmic tail spares mucosal CD 4 cells but does not prevent disease progression. J Virol. 2013;87(3):1528-1543.

8. Breed MW, et al. Elite control, gut CD4 T cell sparing, and enhanced mucosal $\mathrm{T}$ cell responses in Macaca nemestrina infected by a simian immunodeficiency virus lacking a gp 41 trafficking motif. J Virol. 2015;89(20):10156-10175.

9. Fultz PN, et al. In vivo attenuation of simian immunodeficiency virus by disruption of a tyrosine-dependent sorting signal in the envelope glycoprotein cytoplasmic tail. J Virol. 2001;75(1):278-291.

10. Sestak K, Conroy L, Aye PP, Mehra S, Doxiadis GG, Kaushal D. Improved xenobiotic metabolism and reduced susceptibility to cancer in gluten-sensitive macaques upon introduction of a gluten-free diet. PLOS ONE. 2011;6(4):e18648.

11. Rozot V, et al. Mycobacterium tuberculosisspecific CD8+ T cells are functionally and phenotypically different between latent infection and active disease. Eur J Immunol. 2013;43(6):1568-1577.

12. Walzl G, Ronacher K, Hanekom W, Scriba TJ, Zumla A. Immunological biomarkers of tuberculosis. Nat Rev Immunol. 2011;11(5):343-354.

13. Geginat J, Sallusto F, Lanzavecchia A. Cytokine-driven proliferation and differentiation of human naive, central memory, and effector memory CD4(+) T cells. J Exp Med. 2001;194(12):1711-1719.

14. Matthews K, et al. HIV-1 infection alters CD4+ memory T-cell phenotype at the site of disease in extrapulmonary tuberculosis. Eur J Immunol. 2012;42(1):147-157.

15. Nakayama K, et al. Imbalanced production of cytokines by $\mathrm{T}$ cells associates with the activation/exhaustion status of memory T cells in chronic HIV type 1 infection. AIDS Res Hum Retroviruses. 2012;28(7):702-714.

16. Wang Z, Zhang Y, Liu H, Salati E, ChirivaInternati M, Lim SH. Gene expression and immunologic consequence of SPAN-Xb in myeloma and other hematologic malignancies. Blood. 2003;101(3):955-960.

17. Christie JD, et al. Variation in the myosin light chain kinase gene is associated with development of acute lung injury after major trauma. Crit Care Med. 2008;36(10):2794-2800.

18. Agarwal N, Lamichhane G, Gupta R, Nolan S, Bishai WR. Cyclic AMP intoxication of macrophages by a Mycobacterium tuberculosis adenylate cyclase. Nature. 2009;460(7251):98-102.

19. Hultquist JF, et al. Human and rhesus APOBEC3D, APOBEC3F, APOBEC3G, and APOBEC3H demonstrate a conserved capacity to restrict Vifdeficient HIV-1. J Virol. 2011;85(21):11220-11234.

20. Contento RL, et al. CXCR4-CCR5: a couple modulating T cell functions. Proc Natl Acad Sci US A. 2008;105(29):10101-10106.

21. Molon B, et al. T cell costimulation by chemokine receptors. Nat Immunol. 2005;6(5):465-471.

22. Kahnert A, Höpken UE, Stein M, Bandermann S, Lipp M, Kaufmann SH. Mycobacterium tuberculosis triggers formation of lymphoid structure in murine lungs. J Infect Dis. 2007;195(1):46-54.

23. Kalsdorf B, et al. HIV-1 infection impairs the bronchoalveolar T-cell response to mycobacteria. Am J Respir Crit Care Med. 2009;180(12):1262-1270. 